\title{
Timely cancer diagnosis and management as a chronic condition: opportunities for primary care
}

\author{
Moyez Jiwa, Christobel M Saunders, Sandra C Thompson, Lorna K Rosenwax, Scott Sargant, Eric L Khong, Georgia K B Halkett, \\ Gloria Sutherland, Hooi C Ee, Tanya L Packer, Gareth Merriman and Hayley R Arnet
}

$\mathrm{O}$ ne in three men and one in four women in Australia will be diagnosed with cancer in the first 75 years of life, overtaking heart disease as the biggest cause of death in some states in Australia. ${ }^{1}$ Although cancer remains the most feared disease, ${ }^{2} 60 \%$ of patients will eventually die of unrelated causes. ${ }^{3,4}$ But "surviving" cancer does not guarantee an acceptable quality of life for either patients or their families.

After treatment, a person may be cured or may experience troublesome sequelae, a recurrence, or the prospect of death. How an individual copes depends not only on the biological characteristics of the cancer and the treatments available, but also the person's social supports, reserves for dealing with uncertainty, and ability to participate in decisions around treatment options, as well as treatment itself. Most people experience some physical, social, economic and psychological sequelae, leading the World Health Organization to include cancer in its list of chronic conditions. ${ }^{5,6}$

As members of a multidisciplinary team, we would like to propose a number of strategies for meeting the cancer challenge. We do not claim the ideas as novel, but they have seldom been framed for debate in this way. We also acknowledge that the issues are complex, there may be gaps in the evidence base, and some of the innovations we propose may require further evaluation and policy debate. We highlight four specific challenges in caring for people with cancer and propose four strategies for overcoming existing limitations, all of which may be addressed in an integrated primary care hub (Box 1).

\section{Cancer as a chronic condition in Australia: current limitations to care of cancer patients}

\section{Strategies for early diagnosis need to be refined}

Screening asymptomatic people for treatable cancers is an established strategy for timely diagnosis of some cancers. We argue that, in primary care, prompt referral of symptomatic people is also important. However, patients may present to their general practitioner with undifferentiated illness, often with a host of other

\section{ABSTRACT}

- One in three men and one in four women in Australia will be diagnosed with cancer in the first 75 years of life.

- The majority will survive the cancer and ultimately die from unrelated causes.

- Many cancer patients and their families will experience some physical, social, economic and psychological sequelae, regardless of the prognosis.

- A recurring theme is that patients are disadvantaged by the lack of coordination of care and their needs are not being adequately met.

- We argue that greater integration of care through a multidisciplinary team of professionals, peer support groups and primary health practitioners functioning within a care hub could offer better practical and psychosocial supportive care for patients and their families.

MJA 2008; 189: 78-82

For editorial comment, see page 59

needs. Symptoms may have aetiology in the physical, psychological and/or social domains. It is up to the GP to selectively refer patients who are most likely to benefit from diagnostic tests. One of the most telling clues that may alert the GP to suspect cancer is deviation from the patient's normal or usual symptoms or behaviour. While the Australian model of fee-for-service offers choice, it does not encourage people to attend the same practice for all consultations. This reduces the scope for significant new symptoms to be fully appreciated.

The experience in the United Kingdom also suggests that published guidelines as the basis for streaming referrals to appropriate specialists has failed to benefit patients. ${ }^{7}$ Effective casefinding warrants appropriate and timely referral of people with "red-flag" cancer symptoms (such as haematuria, postmenopausal bleeding or unexplained iron deficiency anaemia). While there is a growing literature on the positive predictive value of some red-flag

1 Limitations to current cancer care in Australia and strategies for improvement

Current limitations

- Strategies for early diagnosis need to be refined

- Care is hampered by lack of continuity and coordination

- Supportive care is fragmented or missing

- Minority groups are not served well

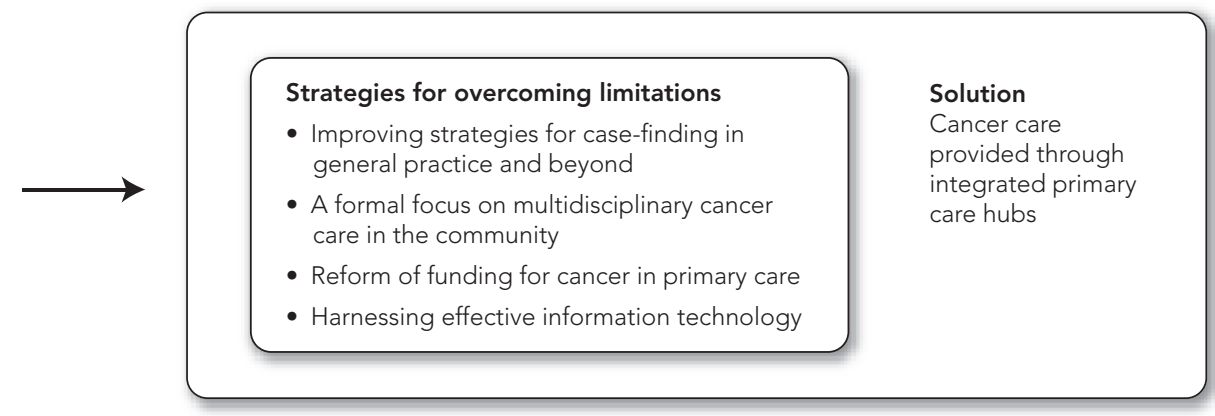




\section{CHRONIC DISEASE - FOR DEBATE}

cancer symptoms, ${ }^{8}$ there are few recognised effective strategies to implement this evidence.

\section{Care is hampered by lack of continuity and coordination}

People with serious illnesses have consistently expressed the need for continuity from a care provider who is available, is genuinely interested in them, provides emotional support, and takes the time to understand and communicate with them and important others. ${ }^{3,9}$ We argue that modern cancer treatment should not fail in this regard. People now wish to have a say in what happens to them - in what bears upon their treatment and how they live their life during and after treatment. ${ }^{10}$ It remains a challenge to guide patients who may be desperate because of a bleak prognosis to accept evidence-based approaches rather than seek "miracle" cures. Information and care are largely delivered in specialist centres, which are seldom designed to accommodate the variety of patients' cultural, familial or social backgrounds. Arguably, the coordination of care could be better managed by a primary care provider with an understanding of the patient's social context.

Landmark qualitative research on living with a chronic condition highlights three aspects that the patient must manage: the disease and its symptoms, the emotional consequences, and the impact on daily function and life roles. ${ }^{11}$ With acute care focused on the first aspect, even when clinical guidelines recommend otherwise, systems of care do not ensure coordination or continuity. For example, evidence-based interventions for fatigue are available, but rarely form part of usual care. Likewise, emotional concerns are recognised, but rarely approached systematically or comprehensively.

\section{Supportive care is fragmented or missing}

Levels of psychological stress experienced by partners of patients with cancer may be similar to those experienced by the patients themselves, or sometimes even higher. ${ }^{12,13}$ Many couples confront cancer as a team, and adjustment to cancer by one partner affects the other. ${ }^{14}$ However, spouses receive minimal support from the medical team, ${ }^{15}$ and it is well documented that partners deserve greater support and care than they currently receive, despite not being the identified patient.

Children of older patients with cancer are often at a loss to know how to respect their parent's privacy while at the same time being involved in the treatment plan and understanding the nature of the illness, for their own and their parent's psychological health. Often there are supportive friends who would also like to be involved by providing assistance in day-to-day care. All too often, the role of significant others and the wider impact of the diagnosis on them receives little recognition.

\section{Minority groups are not served well}

Finally, there is a need to adapt programs to ensure they also meet the needs of minority groups, which are often particularly vulnerable. It remains an indictment of our health care system that Indigenous Australians experience later cancer diagnosis, lower 5year survival, higher mortality rates than non-Indigenous Australians, and lower participation in screening programs. ${ }^{16-18}$ While there has been a 30\% reduction in cancer mortality rates in Australia over the past two decades, there has been little change in Indigenous cancer mortality rates, and there is a need to reconsider how all aspects of cancer control are managed for this group. ${ }^{19}$
Surprisingly little is understood about the experience of culturally and linguistically diverse (CALD) groups with cancer. The incidence of many cancers is related to lifestyle factors such as diet, physical activity, smoking and environmental exposures. Culture also exerts a profound influence on lifestyles and behaviour and shapes an individual's understanding of illness and his or her willingness to access screening and treatment. However, participation rates in research studies are traditionally low among disadvantaged and ethnic minority groups in Australia. As cancer research often excludes or inadequately represents CALD cancer patients, little is documented about their needs.

Minority groups also include people with non-traditional sexual behaviours, which can increase the risk of developing some cancers. ${ }^{20}$ Such groups also present challenges in relation to routine screening programs. For example, general barriers to cervical cancer screening (eg, feeling uncomfortable or disliking the Pap test, low perceived risk, fear, and access factors) are similar for all women. However, homosexual women are unlikely to attend for contraceptive advice and consequently less likely to be offered Pap tests at routine consultation. An additional barrier to screening in this group may be the incorrect assumption of lower cervical cancer risk. In practice, women who are categorised as homosexual are reported to have roughly equal numbers of male and female sexual partners in their lifetime, and thus their risk of acquiring cervical cancer is similar to that of heterosexual women. $^{21}$

\section{Strategies for overcoming current limitations to care of cancer patients}

We propose four overarching strategies for overcoming current limitations to care of people with cancer (Box 1). These could be implemented through integrated primary care hubs that would provide high-quality integrated and multidisciplinary care for people with cancer and other chronic conditions. Although such hubs could also service the needs of people with other chronic diseases in the community (eg, cardiovascular disease, arthritis or diabetes), the present discussion specifically focuses on cancer patients. Typical cancer care scenarios comparing patient care with or without access to an integrated primary care hub are presented in Box 2.

\section{Improving strategies for case-finding in general practice and beyond}

There is some evidence that early cancer may produce detectable symptoms that are not recognised as such, and thus that prognosis may be improved by more effective case-finding. ${ }^{22}$ Effective tools for alerting GPs to the possibility of cancer among symptomatic patients are needed.

There are also potential benefits to be gained by widening the dragnet of case-finding to incorporate a larger group of primary care providers. For example, pharmacists have been estimated to have as many as 75 million contacts with Australian consumers annually. ${ }^{23}$ The ongoing patient-practitioner relationship enjoyed by many community pharmacists and their clients has been undervalued. Many patients seek over-the-counter remedies for red-flag cancer symptoms, and the opportunity for pharmacists to identify such patients at a stage when the condition is still amenable to treatment is well recognised, despite the fact that 


\section{Typical cancer care scenarios illustrating patient care with or without access to an integrated primary care hub}

Background: At the age of 43 years, Mrs $\mathrm{H}$ was diagnosed with breast cancer. Her husband's work requires him to travel away from home frequently. They have two children, a girl aged 17 (in her final year of school) and a boy aged 16 .

When Mrs $\mathrm{H}$ noticed a lump in her breast, she immediately consulted her general practitioner. The following are two typical scenarios for how subsequent events may have unfolded.

\section{Scenario 1: non-integrated cancer care}

The GP referred her to the local radiology clinic for diagnostic assessment. Within a week after the diagnosis was confirmed, she was referred to and assessed by a highly regarded breast cancer surgeon at his rooms. The following week, she had breastconserving surgery with axillary clearance after cancer cells were detected in a sentinel node biopsy.

Mrs $\mathrm{H}$ attended a breast clinic, as instructed, for a series of chemotherapy treatments, followed by radiotherapy treatments. These left her feeling nauseated and tired. Each attendance for treatment took up a large part of the day - travelling, waiting to be seen, and undergoing the treatment itself. Mrs $\mathrm{H}$ declined the offer to access the hospital counsellor, as she was worried about the cost, the time and the effort required to get there. Unfortunately, after the treatment, she developed lymphoedema, which required compression bandages.

It was not until after the treatment had finished that she went back to see her GP. The GP arranged for her to see a counsellor and a physiotherapist, who worked independently to address her specific needs within their professional domains.

The wider impact of the diagnosis included her anxiety about the risk of cancer in other family members and her inability to function as a homemaker because of fatigue following therapy. No agency addressed the impact of the cancer on her family as a unit.

\section{Scenario 2: integrated cancer care}

The GP referred her for testing to the local radiology clinic and subsequently as a private patient to a reputable breast cancer surgeon. Her GP, with her consent, also alerted a cancer care coordinator, who took a full social history and identified several issues causing anxiety: the family had substantial financial commitments, and their mortgage repayments relied on the income of both partners; Mrs H's father had died of cancer 6 months earlier, and although Mrs $\mathrm{H}$ was very close to her frail mother, she didn't want to make her mother anxious by telling her about the diagnosis at this stage.

Mrs $\mathrm{H}$, with her family, was supported to actively address a plethora of concerns, including financial issues (which meant her daughter could stop her casual night-time work to concentrate on her studies); her need for support from her partner; and negotiations with her son around expectations and limit-setting. Her mother became involved in the family case conferences, and seemed to develop a new sense of purpose and energy around supporting her daughter and the family.

Mrs $\mathrm{H}$ was also introduced to a cancer support group. She appreciated practical support with transport to attend treatment, discussion and education about cancer (including familial risk factors for cancer and lymphoedema management), advice on diet and exercise, and validation of her emotions relating to the cancer. pharmacists' potential impact on case detection is underexplored. $^{24}$

\section{A formal focus on multidisciplinary cancer care in the community}

Of growing importance is the involvement of primary care providers during active treatment, as oral chemotherapeutic and biological agents are developed and inpatient stays for surgery shorten. ${ }^{25}$ GPs have a role in managing acute toxicities during treatment or perhaps administering chemotherapy in close consultation with the specialist team. Community pharmacists could also become more involved in advising patients about symptom management, as well as supporting patients with sometimes complex adjuvant treatment regimens with potentially adverse effects. ${ }^{26}$

Occupational therapists, physiotherapists, community nurses, psychologists, counsellors, social workers and nutritionists also have a valued role to play in helping patients manage the symptoms, emotional consequences and impact on their daily life roles. Examples include rehabilitation to allow patients to maintain or regain independence in self-care; modifications to homes; selfmanagement programs for common cancer symptoms (such as fatigue, psychosocial distress and anxiety); pain management techniques; lymphoedema care; graded exercise programs; carer education; and nutritional advice. While these interventions are included in algorithms for best practice (such as the National Comprehensive Cancer Network practice guidelines in oncology: cancerrelated fatigue ${ }^{27}$ ), the mechanisms for ensuring patient access to such services do not yet exist. Nor do mechanisms to allow multiple professionals to communicate with each other and with the patient.

Primary care also plays an important role for cancer patients living in rural and remote areas. The difficulties of geographical isolation are exacerbated for many Indigenous Australians, who are subject to additional sociocultural and economic factors that may profoundly influence survival. ${ }^{28}$ Firstly, rural and remote patients have difficulty travelling to cancer treatment centres or obtaining comprehensive treatment locally. ${ }^{29}$ Secondly, cultural and religious differences may exacerbate the reticence to accept conventional therapies, particularly if a preference for traditional healing practices is ignored. ${ }^{30}$ There may also be heightened concerns about how a patient's immediate and extended family will cope with the illness and treatment, especially if after care includes practices that are at odds with the patient's deeply held cultural ideas, concerns and expectations. ${ }^{30}$

\section{Reform of funding for cancer in primary care}

Organisational and financial structures are essential to support high-quality care. To increase involvement of primary care in population-based screening programs and to engage GPs in particular, the payment structure - which currently rewards service to individual symptomatic patients rather than opportunistic counselling of asymptomatic people - needs to be reviewed. Enhanced Primary Care item numbers remunerate provider conferencing for care planning, but these financing arrangements do not enable direct access to health professionals other than medical practitioners, even when other health care providers may be better placed to address many aspects of cancer care.

Private health care organisations have particular importance in the Australian context, but access to coordinated multidisciplinary care for all people with chronic conditions, regardless of their 
income, requires investment of public funds. Ineffective multidisciplinary care at the primary care level will limit our response to the challenges we have highlighted here. In its 2006-07 federal budget, the Australian Government allocated unprecedented resources to self-management as one of five key strategies to cope with the existing epidemic of chronic conditions. ${ }^{31}$ However, little innovation is occurring in this area of cancer care. Reform is essential to remedy the federal-state divide in health care provision, which results in fragmented and disconnected care. ${ }^{32}$ Recognition is overdue that providers of primary care extend beyond medical practitioners to a wider spectrum of practitioners in the community, including patients themselves and their families. ${ }^{33}$ To this end, it is essential that anything that helps people cope with their cancer is recognised, acknowledged and supported.

\section{Harnessing effective information technology}

It is important to help health care providers offer patients advice and information that is consistent with current evidence and for all health providers to have access to that information. Information technology offers the best prospect of enabling practitioners to make referrals, share data and refer to the latest evidence on an interactive basis. But such innovations will only succeed if they are designed for and with practitioners and allow the users to address patients' multiple needs without feeling burdened by the technology. Such developments may significantly improve compliance with guidelines, such as those published by the Royal Australian College of General Practitioners. ${ }^{34}$ In Australia, shared care arrangements, particularly those emphasising "informational continuity" of care so that primary care professionals are kept abreast of how their patients are being managed by a multidisciplinary team, offer prospects for improved patient care. ${ }^{35}$ Schemes that hold particular promise are those in which practitioners in different sectors have access to the same data on patients managed between them, through either patient-held records or shared access to computer databases. ${ }^{36}$

\section{One way forward: integrated primary care hubs for cancer care}

The evidence shows that collaboration between care providers, coordination of care and patient involvement contribute to better quality health care. ${ }^{37}$ Australian commentators are now touting a move away from the biomedical practice model to a communitybased, primary care practice model, acknowledging that it will deliver better health outcomes at lower cost than a health system that focuses on hospital medical practice. ${ }^{33}$ A recurring theme has been that patients are disadvantaged by the lack of coordination of care.

A possible remodelling of health care for people with cancer, and potentially other chronic conditions, is summarised in Box 3. The acute phase of cancer treatment would continue to be managed by specialists, given the need to apply costly, technologically intensive and scarce treatment modalities. However, primary care providers could have more contact with patients during the active treatment phase. We argue that greater involvement of professionals in the community, functioning within a care hub, could offer better practical and psychosocial support than patients receive under the present system. At the centre of the hub would be the patient, his or her carer, and wider family. Within the hub, the GP would remain closely involved, but after diagnosis, the

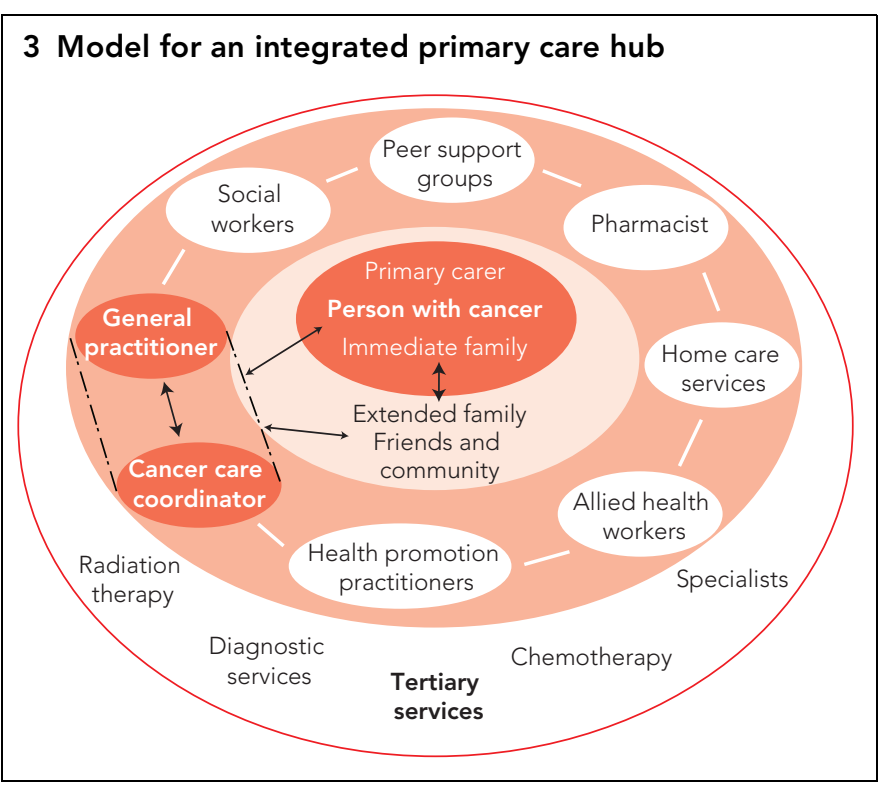

support and coordination role could also be undertaken by another health professional acting as the patient's advocate and with the authority to harness input from a range of paramedical, social and welfare agencies.

A critical element of the hub would be a strong link to peer support services so that patients could benefit from the experience of survivors and access informed advice about treatment choices. Such groups could also provide practical help, such as transport, childcare, housework or supporting the person through treatment. Cancer coordinators could facilitate multidisciplinary case conferencing for complex cases, using teleconferencing and videoconferencing as necessary. All members of the team would be offered training in cultural sensitivity, and cultural advisors and interpreters would be used as appropriate. Ongoing interprofessional development of team members in relation to cancer care would supplement lessons from case conferencing.

By integrating services with reference to the four strategies described in Box 1 and addressing unmet need as described in Box 2, integrated primary care hubs would also provide an excellent opportunity to link to broader efforts at primary and secondary prevention of cancer through the inclusion of public health practitioners. Cancer has both environmental and genetic causes, and so a diagnosis of cancer in one member of a family could provide the opportunity to assess cancer risk among other family members and in the wider community. Encouragement of screening, support for smokers to quit smoking, advice around healthy lifestyles and other similar initiatives could be seen as ways of reducing risk for developing cancer and improving mental and physical wellbeing.

\section{Acknowledgements}

We would like to acknowledge Professor David Currow and Dr Simon Towler for cogent and constructive review of our manuscript. With special thanks to Bob Tomlins for help with the figure in Box 3.

\section{Competing interests}

None identified. 


\section{Author details}

Moyez Jiwa, MD, MRCGP, FRACGP, Professor of Health Innovation ${ }^{1}$ Christobel M Saunders, FRCS, FRACS, Professor of Surgical Oncology ${ }^{2}$ Sandra C Thompson, FAFPHM, PhD, Associate Professor ${ }^{1}$

Lorna K Rosenwax, BAppSc(OT), MSc, PhD, Head, School of Occupational Therapy

Scott Sargant, BPharm, MPS, Adjunct Lecturer, School of Pharmacy ${ }^{1}$

Eric L Khong, MB BS, PGradDipPrimHlthCare, FRACGP, Senior Lecturer $^{3}$

Georgia K B Halkett, BMedRad(Hons), FIR, PhD, National Breast Cancer Foundation David Jones Postdoctoral Research Fellow ${ }^{4}$

Gloria Sutherland, BAppSc, PGradDipHIthEd\&Prom, MSc, Senior Project Coordinator, Bowel Cancer Screening Implementation Team ${ }^{5}$

Hooi C Ee, MB BS, FRACP, PhD, Head 6

Tanya L Packer, BSc(OT), MSc, PhD, Professor and Director, School of Occupational Therapy ${ }^{1}$

Gareth Merriman, BAppSc(Psych) MPsych, PhD, Program Leader, Director of Health Promotion and Sexology, and Senior Lecturer ${ }^{1}$ Hayley R Arnet, BHSc(Pod), PGradDipHIthlnform, Project Officer ${ }^{4,5}$ 1 Faculty of Health Sciences, Curtin University of Technology, Perth, WA. 2 School of Surgery and Pathology, University of Western Australia, Perth, WA.

3 School of Nursing, Midwifery and Postgraduate Medicine, Edith Cowan University, Perth, WA.

4 Western Australian Centre for Cancer and Palliative Care, Curtin University of Technology, Perth, WA.

5 Western Australian Cancer and Palliative Care Network, WA

Department of Health, Perth, WA.

6 Department of Gastroenterology and Hepatology, Sir Charles Gairdner Hospital, Perth, WA.

Correspondence:m.jiwa@curtin.edu.au

\section{References}

1 Australian Bureau of Statistics. Causes of death, Australia, 2006. Canberra: ABS, 2008. (ABS Cat. No. 3303.0.) http://abs.gov.au/Ausstats/ abs@.nsf/e8ae5488b598839cca25682000131612/2093da6935db 138 fca2568a9001393c9! OpenDocument (accessed Apr 2008).

2 Borland R, Donaghue N, Hill D. Illnesses that Australians most feared in 1986 and 1993. Aust J Public Health 1994; 18: 366-369.

3 Australian Institute of Health and Welfare and Australasian Association of Cancer Registries. Cancer in Australia 2001. Canberra: AlHW, 2004. (AlHW Cat. No. CAN 23; Cancer Series No. 28.)

4 Australian Bureau of Statistics. Australian social trends, 2004. Canberra: ABS, 2004. (ABS Cat. No. 4102.0.) http://www.abs.gov.au/Ausstats/ abs@.nsf/0/8EF93FC73F152D78CA256E9E002828EB?Open (accessed Jun 2007).

5 Cancer survivors: living longer, and now, better. Lancet 2004; 364: 21532154.

6 World Health Organization. 58th World Health Assembly approved resolution on cancer prevention and control. http://www.who.int/cancer/ eb1143/en/index.html (accessed Nov 2007).

7 Harris MF, Zwar NA. Care of patients with chronic disease: the challenge for general practice. Med J Aust 2007; 187: 104-107.

8 Hamilton W, Peters TJ, Round A, Sharp D. What are the clinical features of lung cancer before the diagnosis is made? A population based casecontrol study. Thorax 2005; 60: 1059-1065.

9 Curtis JR, Wenrich MD, Carline JD, et al. Patients' perspectives on physician skill in end-of-life care: differences between patients with COPD, cancer, and AIDS. Chest 2002; 122 (1): 356-362.

10 Radley A. "Cases" and voices: the changing agenda for doctors and their patients. Postgrad Med J 2002; 78: 701-702.

11 Corbin J, Strauss A. Managing chronic illness at home: three lines of work. Qual Sociol 1985; 8: 224-247.

12 Baider L, Koch U, Esacson R, De-Nour AK. Prospective study of cancer patients and their spouses: the weakness of marital strength. Psychooncology 1998; 7: 49-56.
13 Harden J, Schafenacker A, Northouse L, et al. Couples' experiences with prostate cancer: focus group research. Oncol Nurs Forum 2002; 29: $701-$ 709.

14 Northouse L, Templin T, Mood D. Couples' adjustment to breast disease during the first year following diagnosis. J Behav Med 2001; 24: 115-136.

15 Harrison J, Haddad P, Maguire P. The impact of cancer on key relatives: a comparison of relative and patient concerns. Eur J Cancer 1995; 31A: 1736-1740.

16 Condon JR, Cunningham C, Barnes T, et al. Cancer diagnosis and treatment in the Northern Territory: assessing health service performances for Indigenous Australians. Intern Med J 2006; 36: 498-505.

17 Australian Institute of Health and Welfare. Cervical screening in Australia 2001-2002. Canberra: AlHW, 2004. (AlHW Cat. No. CAN 22; Cancer Series No. 27.)

18 Australian Institute of Health and Welfare. BreastScreen Australia monitoring report 2001-2002. Canberra: AlHW, 2005. (AlHW Cat. No. CAN 24; Cancer Series No. 29.)

19 Lowenthal RM, Grogan PB, Kerrins ET. Reducing the impact of cancer in Indigenous communities: ways forward. Med J Aust 2005; 182: 105-106.

20 Anderson JS, Vajdic C, Grulich AE. Is screening for anal cancer warranted in homosexual men? Sex Health 2004; 1: 137-140.

21 Matthews AK, Brandenburg DL, Johnson TP, Hughes TL. Correlates of underutilization of gynaecological cancer screening among lesbian and heterosexual women. Prev Med 2004; 38: 105-113.

22 Corner J, Hopkinson J, Fitzsimmons D, et al. Is late diagnosis of lung cancer inevitable? Interview study of patients' recollections of symptoms before diagnosis. Thorax 2005; 60: 314-319.

23 Howarth H, Peterson, G, Jackson S, et al. Report of health promotion and screening activities by community pharmacists 2005. Community Pharmacy Research Support Centre. http://www.guild.org.au/uploadedfiles/ Research_and_Development_Grants_Program/Projects/2002-508_ fr4.pdf (accessed Jun 2007).

24 Connelly D. Community pharmacists can play a key role in raising awareness of bowel cancer. Pharm J 2007; 278: 580.

25 DeMario MD, Ratain MJ. Oral chemotherapy: rationale and future directions. J Clin Oncol 1998; 16: 2557-2567.

26 Riechelmann RP, Tannock IF, Wang L, et al. Potential drug interactions and duplicate prescriptions among cancer patients. J Natl Cancer Inst 2007; 99: 592-600.

27 National Comprehensive Cancer Network. NCCN practice guidelines in oncology: cancer-related fatigue. http://www.nccn.org/professionals/ physician_gls/PDF/fatigue.pdf (accessed Nov 2007).

28 Cunningham J. Diagnostic and therapeutic procedures among Australian hospital patients identified as Indigenous. Med J Aust 2002; 176: 5862.

29 Coory M, Thompson A, Ganguly I. Cancer among people living in rural and remote Indigenous communities in Queensland. Med J Aust 2000; 173: 301-304.

30 National Aboriginal and Torres Strait Islander Health Council. National Strategic Framework for Aboriginal and Torres Strait Islander Health: framework for action by governments. Prepared by NATSIHC for the Australian Health Ministers' Conference. Canberra: NATSIHC, 2003.

31 Jordan JE, Osborne RH. Chronic disease self-management education programs: challenges ahead. Med J Aust 2007; 186: 84-87.

32 Mann L. From "silos" to seamless healthcare: bringing hospitals and GPs back together again. Med J Aust 2005; 182: 34-37.

33 Doggett J. A new approach to primary care for Australia. Sydney: Centre for Policy Development, 2007. (Occasional Paper No. 1.)

34 Royal Australian College of General Practitioners. Guidelines for preventive activities in general practice. 6th ed. Melbourne: RACGP, 2005. http:// www.racgp.org.au/guidelines/redbook (accessed Sep 2007).

35 Haggerty JL, Reid RJ, Freeman GK, et al. Continuity of care: a multidisciplinary review. BMJ 2003; 327: 1219-1221.

36 Hickman M, Drummond N, Grimshaw J. A taxonomy of shared care for chronic disease. J Public Health Med 1994; 16: 447-454.

37 Canadian Health Services Research Foundation. Interdisciplinary teams in primary healthcare can effectively manage chronic illnesses. Evidence Boost for Quality; Sep 2005. http://www.chsrf.ca/mythbusters/pdf/ boost3_e.pdf (accessed Jan 2008).

(Received 23 Jul 2007, accepted 30 May 2008) 